\title{
Oral Pigmentation as a Sign of Addison's Disease: A Brief Reappraisal
}

\author{
Alessandro Lanza ${ }^{1,2}$, Inam Heulfe ${ }^{3}$, Letizia Perillo ${ }^{2}$, Antonio Dell'Ermo ${ }^{2}$ and Nicola Cirillo *,1 \\ ${ }^{1}$ Regional Center on Craniofacial Malformations-MRI, ${ }^{2}$ Department of Oral Sciences, $I^{\text {st }}$ School of Medicine and \\ Surgery, II University of Naples, 80138 Naples, Italy \\ ${ }^{3}$ Bristol Dental Hospital, BS1 2LY Bristol, UK
}

\begin{abstract}
Pigmented lesions are frequently found in the oral cavity. Their overlapping clinical features should prompt the physician to carefully evaluate the patient's medical history for a differential diagnosis. Oral pigmentations may represent physiological changes or be a manifestation of more serious diseases. Here, we describe a patient who was affected by brown maculae involving the perioral region and oral cavity. The diagnostic challenge of Addison's disease along with a brief review on the topic is then presented.
\end{abstract}

Keywords: Addison's disease, oral pigmentation, cortisol, ACTH.

\section{INTRODUCTION}

Oral pigmentations may be focal, diffuse or multifocal, and macular or raised. Diffuse and multifocal pigmentation is attributable to an increased synthesis of melanin called melanosis caused by systemic or pharmacological factors. Oral melanotic maculae may represent intraoral freckles, post-inflammatory pigmentation or maculae associated to systemic diseases. When melanotic maculae are found excessively in an oral and perioral distribution, Peutz-Jeghers syndrome or Addison's disease should be considered. A critical evaluation of the patient's medical history, intraoral and extraoral examination and laboratory tests are necessary to make a differential diagnosis; in addition a biopsy should be performed in doubtful cases [1]. This case report presents a patient affected by oral pigmentation and peculiar symptomatology that directs the investigation towards Addison's disease. In fact a primary involvement of adrenal glands leads to a reduction or lack (very rare) of the main adrenal hormones, causing many signs and symptoms such as nausea, vomiting, abdominal pain, anorexia, weight loss, fatigue and postural hypotension [2]. Furthermore, adrenal failure generates an activation of the pituitary gland leading to an increased release of adrenocorticotropin (ACTH) and melanocyte-stimulating hormone (MSH) as part of a negative feedback mechanism. Hypersecretion of ACTH and other pro-opiomelanocortin derived peptides stimulate melanocytes in the skin and mucosa via $\alpha$-MSH receptor (MC1) inducing skin and oral mucosa pigmentation [3].

\section{CASE PRESENTATION}

A 56-year-old woman was admitted with dark brown maculae at the vermillion lip (Fig. 1a). Previously, her general physician had excluded serious diseases and did not request any additional tests. Extraoral examination showed browny pigmentation of two month's duration, affecting the perioral sites; intraoral examination demonstrated bilateral

*Address correspondence to this author at the Department of Stomatology, Second University of Naples, Via Luigi de Crecchio, 7, 80138, Naples, Italy; Fax: +39 0815665500; E-mail: cirillo.sun@libero.it involvement of the buccal mucosa, gingival, oral mucosa of the lower lips and alveolar mucosa (Fig. 1b). In addition, the patient presented with a history of hypotension, anorexia, abdominal pain, nausea and vomiting and weight loss. Our major diagnostic doubt was between diagnosing diffuse oral maculae due to melanosis or more serious systemic diseases associated to oral diffuse pigmentation such as Peutz-Jeghers syndrome or Addison's disease. Therefore, we requested additional laboratory tests: blood chemistry, blood pressure, colonoscopy, ultrasonography and MRI of the adrenal glands. Colonoscopy did not reveal intestinal polyps. In addition to low blood pressure $(100 \backslash 60 \mathrm{mmHg})$, blood chemistry tests revealed low plasma cortisol associated to hyponatraemia $(125 \mathrm{mEq} / \mathrm{l})$ and hyperkalaemia $(5 \mathrm{mEq} / \mathrm{l})$. Furthermore, a short synacthen test (short corticotrophin test) was requested to confirm adrenal insufficiency, in which impaired response to adrenocorticothropin hormone was found $(250 \mathrm{pg} / \mathrm{ml}$; normal values $=6-60 \mathrm{pg} / \mathrm{ml})$. Ultrasonography and MRI of the adrenal glands showed absence of any masses or swellings that could mimic a neoplasm producing ACTH or haemorrhages (not shown). On the basis of the above findings, a diagnosis of Addison's disease was made. The patient was then referred to the endocrinology division where he was managed for a correct therapy of the disease.

\section{DISCUSSION}

Addison's disease is a primary adrenal failure caused by infiltrative or autoimmune processes. Tuberculosis, various fungal infections, metastases or haemorrhages represent the main infiltrative causes; several autoimmune processes can lead to adrenal insufficiency affecting exclusively the adrenal glands or be part of a more complex inherited autoimmune polyglandular syndrome (APS).

An autoimmune pathogenesis was supposedly due to the finding of circulating autoantibodies against adrenocortical cells in patients' serum samples. Furthermore primary adrenal insufficiency may also be caused by disorders in the main enzymes involved in steroidogenesis or by X-linked adrenoleukodistrophy, an inherited pathology characterized 
by a mutation in ABCD1 gene that may induce death of adrenocortical cells $[4,5]$.

(a)

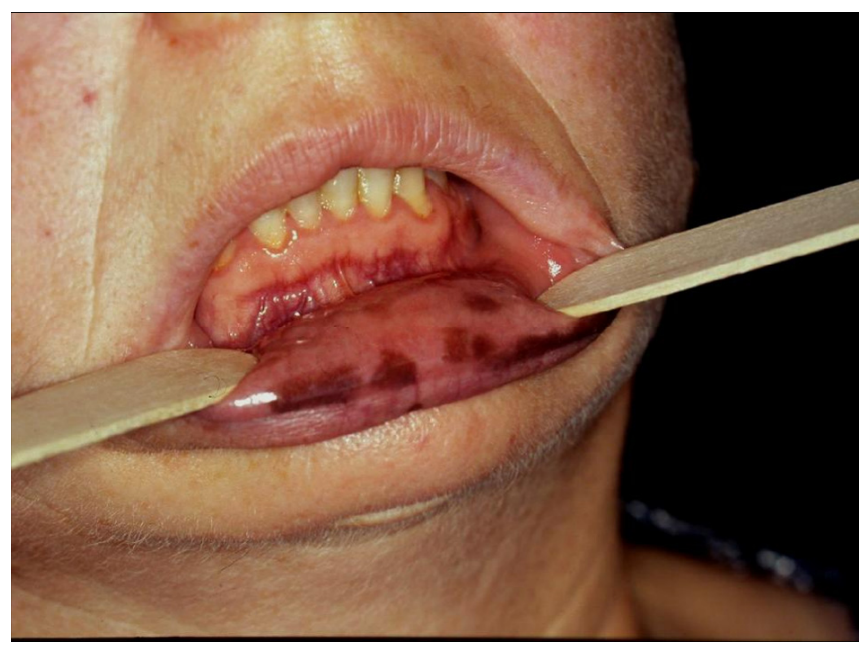

(b)

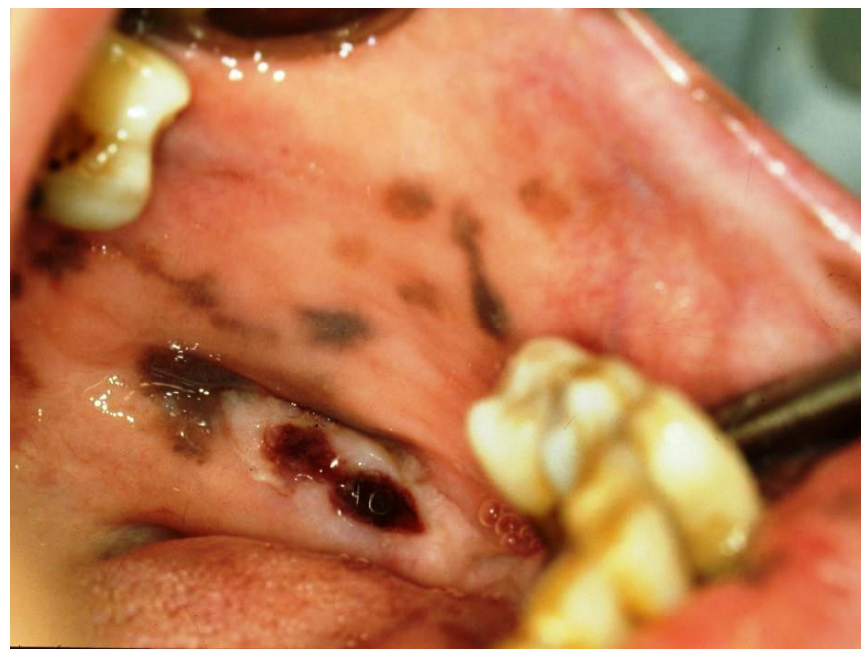

Fig. (1). (a) Dark brown maculae affect alveolar and labial mucosa. The anterior vermillion of the inferior lips is also characterized by diffuse pigmentation; (b) Multifocal pigmentations involving buccal and edentulous alveolar ridge mucosa.

The adrenal cortex is the principal target organ for ACTH that has a key role in the pathogenesis of oral pigmentation acting as the major regulator for cortisol and adrenal androgen. ACTH is also essential for aldosterone biosynthesis, although aldosterone secretion is primarily under the control of the renin-angiotensin axis $[6,7]$.

The biologic activity of ACTH resides in the N-terminal portion, with the first 24 amino acids necessary for maximal activity. ACTH interacts with specific high affinity cell membrane receptors (melanocortin receptor 2, MC2), expressed in all adrenocortical cells that couple to G-proteins to stimulate adenylyl cyclase and generate cAMP [3]. The latter activates a cAMP-dependent protein kinase, which stimulates cholesterol ester hydrolase, the key enzyme in the adrenocortical response of steroidogenesis to ACTH [8]. In addition, ACTH increases the uptake of cholesterol from plasma lipoproteins, enhances later steps in the steroidogenesis and has a trophic effect on the adrenal cortex [9].
Glucocorticoids play a key regulatory role on the basal control of the HPA axis and pituitary gland and the presence of a direct glucocorticoid negative feedback is crucial for the attenuation of the ACTH secretory response. This physiological feedback mechanism may not be triggered because of a primary dysfunction of the Adrenal glands and ACTH blood level remains high as a direct consequence of low serum cortisol. Increasing ACTH and other proopiomelanocortin-derived peptides stimulate the melanocytes via the skin and mucosa $\alpha$-MSH receptor (MC1) causing skin and oral mucosa hyperpigmentation (Fig. 2) [10-12]. Lack of cortisol main signs and sympthoms are: fatigue, lack of energy, anorexia, myalgia, fever, anaemia, lymphocitosis, eosinophilia, hypercalcaemia, increased thyroid stimulating hormone.

Aldosterone, the main mineralcorticoid hormone plays an important role in water and salt balance and in blood pressure regulation. Its reduction accounts for hyponatraemia, hyperkalaemia and salt craving. Instead, dehydroepiandrosterone is a precursor of main sexual-hormones and regulates secondary male sex characteristics. Decreasing dehydroepiandrosterone production leads to androgen deficiency visible clinically as loss of axillary and pubic hair, dry skin, reduced libido and depression (Table 1) [13].

One of the most important signs of Addison's disease is cutaneous and mucosal hyperpigmentatations related to ACTH melanogenesis action. Pigmentation can be homogeneous or blotchy, it does not depend on gender or race. It may involve skin, oral cavity, conjunctiva and genitalia. It is more evident in areas exposed to the sun and under mechanical stimulation: palmar creases, knuckles, flexural areas, areolas of nipples, scars and genital mucosa. Brown patches of gingival, vermillion border of the lips, buccal mucosa, palate and tongue may represent the first signs of Addison's disease [14]. In the evaluation of a patient affected by diffuse and multiple pigmentations the physician has to critically consider the medical history, dental history, extraoral and intraoral examinations requesting, adjunctive laboratory tests to make a diagnosis of systemic involvement. Unique clinical aspects of pigmentation are not enough to reach a correct diagnosis because oral maculae are very similar in several diseases.

However onset and duration of lesions, association with skin pigmentation or/and other mucosal involvement, location and symmetry of the lesions should be considered in addition to laboratory investigations [1]. The physician has to differentiate between pigmentation associated with the physiological and pathological conditions. Oral physiological pigmentations are generally multifocal, symmetric, persistent and race inherited. They are a dark-brown band most frequently affecting the marginal gingiva. They may mimic Addison's pigmentation as clinical features are overlapping. Laboratory investigations should be conducted to exclude more serious diseases [15]. The patient's dental history was negative for trauma, surgical injury or previous inflammatory disease like gingival lichen planus. Consequently oral maculae affecting the patient, which did not represent postinflammatory pigmentation, appeared as a mucosal reaction due to injury. The absence of a history of smoking induced us to exclude smoking melanosis although this last condition presents with diffuse brown-black maculae similar to diffuse 


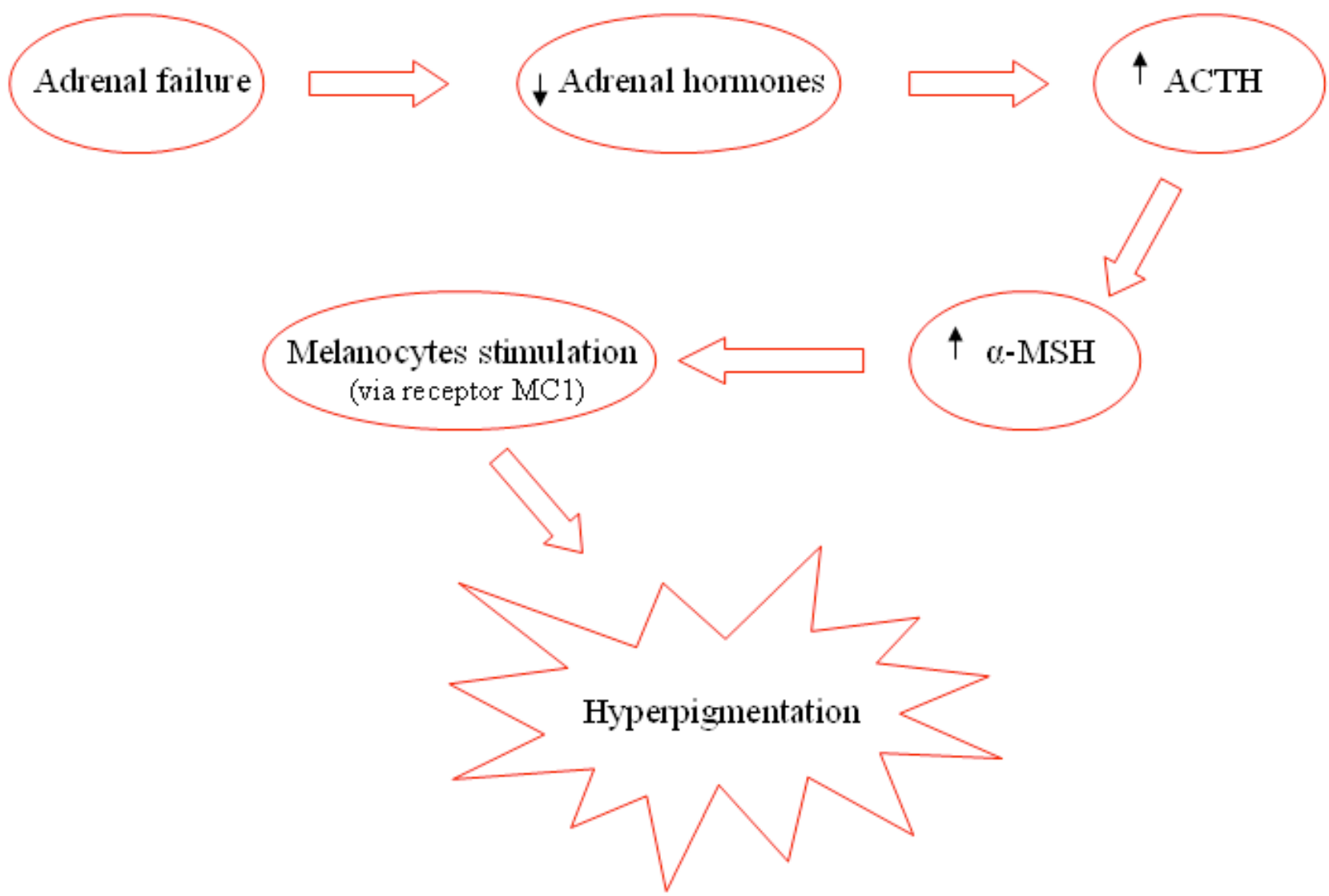

Fig. (2). Mechanism underlying hyperpigmentation in Addison's disease. A primary adrenal failure induces a reduction of the main adrenal hormones, the lack of which triggers an increasing production of adrenocorticotropin (ACTH) by pituitary gland as a part of a feedback mechanism. Increase of ACTH derived peptides stimulates the melanocytes via the skin and mucosa a-MSH receptor (MC1) causing skin and oral mucosa hyperpigmentation.

pigmentation of Addison's disease [16]. Anamnesis was negative for administering of drugs and exposure to heavy

Table 1. Main Clinical and Laboratory Features of Addison's Disease

\begin{tabular}{|c|c|}
\hline Signs and Symptoms & Laboratory Findings \\
\hline \hline Muscle weakness and fatigue & Hypoglycemia \\
\hline Weight loss and decreased appetite & Hyponatraemia \\
\hline Hyperpigmentation & Hyperkalemia \\
\hline Salt craving & Eosinophilia \\
\hline Nausea, diarrhea or vomiting & Lymphocytosis \\
\hline Muscle or joint pains & Low blood pressure \\
\hline Irritability Metabolic acidosis & - \\
\hline Depression & - \\
\hline Weakness & - \\
\hline Dehydration & - \\
\hline Headache, & - \\
\hline Sweating, & - \\
\hline Changes in mood and personality & - \\
\hline
\end{tabular}

The table includes the main signs and symptoms and laboratory findings of this disease. metal vapours so we were able to exclude two frequent causes of oral pigmentation such as drug induced and heavy metal pigmentations. They are further characterized by diffuse or linear brown staining affecting the attached gingiva and palate caused by deposits of drug agglomerates in the bone and tooth roots; the latter is characterized by a black line along the gingival margin [17].

Amalgam tattoo represents the most frequent pigmentation of the oral cavity but the absence of amalgam restorations and increased radiopacity on OPT led us to discard this clinical hypothesis. Oral melanotic maculae are focal pigmented brown lesions similar to epheledes and characterized by a high prevalence in women. Melanotic maculae have been described as occurring predominantly on the vermilion of the lips as patient's pigmentations. They are flat and mostly smaller than $1 \mathrm{~cm}$ and characterised by a focal increase in melanin production not accompanied by the increase in number of melanocytes [18]. However anorexia, fatigue, weight loss and hypotension presented by the patient demonstrated a systemic involvement and, although clinical features were overlapping we excluded a simple melanosis from the differential diagnosis.

Oral naevi and melanomas may also mimic oral Addisonian pigmentation. Oral intramucosal naevus appears as a brown coloured lesion potentially similar to brown maculae of Addison's patients but they are focal and rarely numerous. Generally oral melanoma appears as one isolated 
brown or black patch with asymmetric and irregular borders. A differential diagnosis with Addison's pigmentation should be considered when clinical features are common to both melanoma and pigmented maculae. The presence of multiple lesions and their clinical aspects in addition to signs and symptoms of systemic involvement excluded a melanoma without the need to perform a biopsy [19].

Accordingly with the patient's history and clinical aspects, the most difficult differential diagnosis was between Addison's disease and Peutz-Jeghers syndrome. PeutzJeghers syndrome is characterized by the presence of early onset pigmented oral maculae, small and multiple spots around the lips and signs and symptoms of abdominal pain, rectal bleeding, diarrhoea and involvement of the conjunctiva, nasal mucosa, skin extremities and rectal mucosa [20]. Diagnosis of Addison's disease is based on the value of early morning serum cortisol, ACTH level and plasma renin activity. Moreover the best way to determine the ability of the adrenal glands to respond to ACTH stimulation is to perform a short corticotrophin test, consisting in measurement of cortisol level after 30-60 minutes of intramuscular or intravenous injection of 250- $\mu$ g ACTH (1-24 cosyntropin). Replacement of insufficient hormones represents the most accepted therapy for Addison's disease. Daily glucocorticoid therapy consists of $15-25 \mathrm{mg}$ of hydrocortisone or 25-37.5 $\mathrm{mg}$ of cortisone acetate in two or three daily doses. Mineralcorticoids deficiency represents a peculiar aspect of primary adrenal insufficiency; reintegration of these hormones should be valued as well. Fludrocortisone is administered in a dose of 0.05-0.2 mg once per day.

Dehydroepiandrosterone's therapy should be reserved for patients whose mood is greatly impaired despite optimimum glucocorticoid and mineralcorticoid replacement. In this case therapy consists of 25-50 $\mathrm{mg}$ of dehydroepiandrosterone administered in the morning [4].

\section{REFERENCES}

[1] Kauzman, Pavone M, Blanas N, Bradley G. Pigmented lesions of the oral cavity. Review, differential diagnosis, and case presentation. J Can Dent Assoc 2004; 70: 682-3.
[2] Løvås K, Husebye ES. Addison's disease. Lancet 2005; 365: 205861.

[3] Mountjoy KG, Robbins LS, Mortrud MT, Cone RD. The cloning of a family of genes that encode the melanocortin receptors. Science 1992; 257: 1248-51.

[4] Arlt W, Allolio B. Adrenal insufficiency. Lancet 2003; 361: 188193.

[5] Martín Martorell P, Roep BO, Smit JW. Autoimmunity in Addison's disease. Neth J Med 2002; 60: 269-75.

[6] Aguilera G. Factors controlling steroid biosynthesis in the zona glomerulosa of the adrenal. J Steroid Biochem Mol Biol 1993; 45: 147-151.

[7] Tait SA, Schulster D, Okamoto M, Flood C. Production of steroids by in vitro superfusion of endocrine tissue: II. Steroid output from whole, capsular and decapsulated adrenals of normal intact, hypophysectomized and hypophysectomized-nephrectomized rats as a function of time of perfusion. Endocrinology 1970; 86: 360-382.

[8] Gwynne JT, Strauss JF 3rd. The role of lipoproteinsin steroidogenesis and cholesterol metabolism in steroidogenic glands. Endocr Rev 1982; 3: 299-329.

[9] Kahri AI, Huhtaniemi I, Salmenpera M. Steroid formation and differentiation of cortical cells in tissue culture of human fetal adrenals in the presence and absence of ACTH. Endocrinology 1976; 98: 33-41.

[10] de Kloet ER, Vreugdenhil E, Oitzl MS, Joels M. Brain corticosteroid receptor balance in health and disease. Endocr Rev 1998; 19: 269-301.

[11] Frim DM, Robinson BG, Pasieka KB, Majzoub JA. Differential regulation of corticotropin-releasing hormone mRNA in rat brain Am J Physiol 1990; 258: E686-92.

[12] Keller-Wood ME, Dallman MF. Corticosteroid inhibition of ACTH secretion. Endocr Rev 1984; 5: 1-2.

[13] Burke CW. Adrenocortical insufficiency. Clin Endocrinol Metab 1985; 14: 947-76.

[14] Nieman LK, Chanco Turner ML. Addison's disease. Clin Dermatol 2006; 24: 276-80.

[15] Ciçek Y, Ertaş U. The normal and pathological pigmentation of oral mucous membrane: a review. J Contemp Dent Pract 2003; 4: 76-86.

[16] Scully C, Felix DH. Oral medicine--update for the dental practitioner: red and pigmented lesions. Br Dent J 2005; 199: 639-45.

[17] Gaeta GM, Satriano RA, Baroni A. Oral pigmented lesions. Clin Dermatol 2002; 20: 286-8.

[18] Kaugars GE, Heise AP, Riley WT, Abbey LM, Svirsky JA. Oral melanotic macules. A review of 353 cases. Oral Surg Oral Med Oral Pathol 1993; 76: 59-61.

[19] Eisen D, Voorhees JJ. Oral melanoma and other pigmented lesions of the oral cavity. J Am Acad Dermatol 1991; 24: 527-37.

[20] Kitagawa S, Townsend BL, Hebert AA. Peutz-Jeghers syndrome. Dermatol Clin 1995; 13: 127-33. 